\title{
Relative Importance and Contribiton of Risk Factors for Diabetic Retinopathy and Macular Edema
}

\author{
Xie J', Fenwick EK ${ }^{1}$, Taouk $Y^{1}$, Nicolaou TE , Wang JJ1,2, Wong TY',3,4 and Lamoureux EL ${ }^{1,3,4 *}$
}

${ }^{1}$ Centre for Eye Research Australia, Royal Victorian Eye and Ear Hospital, University of Melbourne, Australia

${ }^{2}$ Centre for Vision Research, Department of Ophthalmology, Westmead Hospital, Australia

${ }^{3}$ Singapore Eye Research Institute, National University of Singapore, Singapore

${ }^{4}$ Office of Clinical Sciences, Duke-NUS Graduate Medical School, Singapore

\section{Abstract}

Purpose: This study determined the relative importance of risk factors for Diabetic Retinopathy (DR) and Diabetic Macular Edema (DME) and assessed their independent and joint contributions.

Methods: A prospective study of patients with type 2 diabetes was conducted in a tertiary eye hospital in Melbourne, Australia. Patients underwent a comprehensive eye examination and completed standardized administered questionnaires. Blood samples were assessed for glycated haemoglobin $(\mathrm{HbA} 1 \mathrm{c})$; fasting blood glucose; and serum lipids. Dilated fundus photographs were obtained and graded for DR and DME. The relative importance of the risk factors was determined by the independent and common variance explained in DR and DME using Commonality analysis.

Results: A total of 497 patients with type 2 diabetes were included in this analysis. The key risk factors accounted for $44.6 \%$ and $19.5 \%$ of total variances in DR and DME, respectively. The most important risk factor for any DR was duration of diabetes (16.5\% of total explained variance), followed by insulin use (13.1\%), HbA1c (8.5\%), age (3.7\%) and gender $(2.8 \%)$. The most important risk factor for any DME was HbA1c $(9.45 \%)$; followed by age $(5.75 \%)$; duration of diabetes $(3.92 \%)$ and systolic blood pressure (SBP) $(0.36 \%)$. Sixty-one percent of the total explained variance in DR was due to common variance among these risk factors, compared to only $31 \%$ of the in DME.

Conclusion: The total, independent and joint contributions of the key risk factors for DR and DME differ in patients with type 2 diabetes, which suggest that may require specific prevention and intervention strategies for these two diabetic complications.

Keywords: Diabetic retinopathy; Diabetic macular edema; Haemoglobin A1c; Dduration of diabetes; Commonality analysis

\section{Introduction}

Diabetic retinopathy (DR) and diabetic macular edema (DME) are common complication of diabetes and the leading causes of blindness in the working-age population worldwide [1-3]. DME can occur at any stage of DR and affects central visual acuity [4]. It is well recognized that the duration of diabetes, glycated haemoglobin (HbAlc), hypertension, dyslipidemia, and the onset of diabetes are the key risk factors for the prevalence, incidence and progression of DR and DME. Most previous studies assessing the association between these major risk factors and DR/DME use traditional regression methods (e.g., logistic regression models), which do not fully explain the amount of variation in DR/ DME, partly due to the non-inclusion of shared or common variations among the risk factors [5-10]. Other methods, such as hierarchical regression analysis, have been used to determine the amount of variance in the dependent variable that is explained by the independent variable(s). However, the results obtained from hierarchical regression analysis can be influenced by the order of variables entered into the equation.

Furthermore, current estimates of the key risk factors for DR and DME are mostly reported as odds ratios (ORs) or hazard ratios (HRs) and their relative importance are easily misinterpreted by the absolute values of ORs $[11,12]$. In fact, of relevance to clinicians are which variables are more important to focus on and the relative magnitude of their unique and joint contributions to the risk of DR. To date, however, the relative importance and contribution of these risk factors for DR remains unclear [1,5,13-15]. Understanding the relative importance allows prioritization of strategies for DR and DME screening and management.

Commonality analysis (CA) is designed to identify the proportions of variance in the dependent variables to which each of the independent variable attributes uniquely, and also the proportions of variance attributed by various combinations of independent variables [16-19]. CA has been successfully used in the social and behavioural sciences [16-18,20-22], but not in medical diseases. In this study, we assessed the independent and joint contributions of the key risk factors of DR and DME, and determined the relative importance of these risk factors using CA.

\section{Method}

\section{Study Population}

The Diabetes Management Project (DMP) is a longitudinal study of adults with diabetes residing in Victoria, Australia. The methodology

*Corresponding author: Ecosse Lamoureux, Centre for Eye Research Australia 32 Gisborne Street, East Melbourne 3002, Victoria, Australia, Tel: +61 39929 8371; Fax: +61 3 9662-3859; E-mail: ecosse@unimelb.edu.au

Received January 13, 2014; Accepted February 12, 2014; Published February 18,2014

Citation: Xie J, Fenwick EK, Taouk Y, Nicolaou TE, Wang JJ, et al. (2014) Relative Importance and Contribiton of Risk Factors for Diabetic Retinopathy and Macular Edema. J Diabetes Metab 5: 337. doi:10.4172/2155-6156.1000337

Copyright: (c 2014 Xie J, et al. This is an open-access article distributed unde the terms of the Creative Commons Attribution License, which permits unrestricted use, distribution, and reproduction in any medium, provided the original author and source are credited. 
has been described previously [23]. In brief, English-speaking adults aged 18 years or older with type 1 or type 2 diabetes, free of significant hearing and cognitive impairment, and living independently in the community were invited. Patients were primarily recruited from the Royal Victorian Eye and Ear Hospital (RVEEH). This paper reports on the cross-sectional data for patients with type 2 diabetes. All study procedures adhered to the tenets of the Declaration of Helsinki and all privacy requirements were met. Ethical approval for the DMP was provided by the RVEEH Human Research and Ethics Committee $(08 / 815 \mathrm{H})$. Each individual signed a consent form that outlined the aims and methodology of the DMP.

\section{DMP testing protocol}

All examinations were conducted at the Centre for Eye Research Australia (CERA) Melbourne Australia, located at the RVEEH.

\section{Fundus photography}

Two-field $45^{\circ}$ digital non-stereo colour fundus photographs of both eyes were taken from each individual using a non-mydriatic retinal camera (Canon CR6 - 45NM), Canon Inc, Japan, with all images being stored electronically using Digital Healthcare software. DR and DME were assessed using dilated fundus photography. Missing data (fundus images) were encountered in 13 cases (2.5\%).DR severity was classified according to the International Clinical Diabetic Retinopathy Severity Scale into: 1) non-proliferative DR (NPDR) and (2) proliferative DR (PDR). NPDR was further grouped into three progressive stages - mild, moderate and severe - with each stage having defined retinal pathologic signs.

DME severity was classified using the American Academy of Ophthalmology classification as no DME; mild DME; moderate DME; and severe DME (clinical significant macular edema). The presence and severity of DME was also confirmed with OCT images (Stratus) and an ophthalmologist (Co-author TYW).

\section{OCT}

Fast macular scans (right and left eye) with retinal map analysis (fast macular thickness map, retinal thickness/volume tabular), as well as retinal nerve fibre layer scans (retinal nerve fibre layer $3.4 \mathrm{~mm} /$ average retinal nerve fibre layer thickness), were obtained using the OCT Stratus Model 3000 machine (Carl Zeiss Meditec Inc., Dublin, CA, USA).

\section{Questionnaires}

Information associated with lifestyle and psychosocial risk factors were collected using validated questionnaires. We report on data collected only from the general questionnaire on self-reported medical and ocular history; diabetes status and duration; demographics and medication use.

\section{Blood Pressure (BP) measurements}

Each participant underwent a blood pressure (BP) assessment using an automated BP machine, model 5200-103Z (Welch Allyn, $\mathrm{NZ}$ ). The average of two separate measurements was recorded for both systolic (SBP) and diastolic (DBP) measurements.

\section{Blood collection}

Fasting blood ( $\geq 8$ hours) was obtained to assess HbAlc levels, fasting glucose and lipids (total cholesterol [TC], triglyceride [TG], and high-density lipoprotein cholesterol [HDL-C]). Low-density lipoprotein cholesterol [LDL-C] was measured using the Friedewald formula. All biochemical parameters were analysed at Melbourne Pathology, Melbourne, Australia. Fasting plasma glucose and serum lipids were assessed using the Hitachi MODULAR P analyser from Roche Diagnostics (Mannheim, Germany).

\section{Statistical analyses}

Continuous variables are presented as median (interquartile range $[\mathrm{IQR}]$ ) for data with skewed distribution and mean (standard deviation $[\mathrm{SD}]$ ) for normally distributed data, whereas categorical variables are presented as absolute (n) and relative frequencies (\%) and $95 \%$ confidence interval (95\% CI). Differences in continuous variables between participants with and without DR were evaluated by the Mann-Whitney-Wilcoxon test for skewed data, and the t-test for normally distributed data. The proportions between participants with and without DR were compared using the Chi-square test.

The relationships between DR, DME and HbAlc and other risk factors were examined separately using a stepwise logistic regression model and all significant factors therefore were included in the CA. CA was used to decompose the total variation of the model into unique and common variance. Unique variance reflects how much variance a risk factor independently contributes to DR or DME that is not shared with other risk factors. In contract, common variance can provide detailed information that identifies and quantifies the variances explained by a group of risk factors. The relative importance of the risk factors was determined by considering the percentages of the sum of unique and common variance explained. CA was performed in R. A two-tailed p-value $<0.05$ was considered statistically significant.

\section{Results}

\section{Sociodemographic and clinical characteristics of patients with and without DR}

Patients with any DR ( $n=297)$ were younger, had higher use of insulin, and longer duration of diabetes compared to those without $(\mathrm{n}=200$; Table $1, \mathrm{p}<0.001$ for all). One hundred and thirty four $(27.0 \%)$ had proliferative diabetic retinopathy (PDR). Patients with any DR also had higher fasting plasma glucose, HbAlc, and lower HDL cholesterol than those without ( $\mathrm{p}<0.05$ for all). However, there was no difference between the two groups in educational attainment, income, DBP, body mass index (BMI), LDL-C, and triglycerides.

Patients with any DME ( $\mathrm{n}=158)$ were younger, more likely to use insulin and have more comorbidities, longer duration of diabetes, higher fasting plasma glucose, HbAlc and LDL cholesterol level compared to those without any DME (Table S1, $<<0.001$ for all).

\section{Risk factors for DR and DME}

Stepwise logistic regression analysis was used to determine the independent risk factors for DR and DME, separately (Table 2). In the stepwise logistic model, younger age (odds ratio[OR $]=0.95,95 \% \mathrm{CI}$ : 0.92-0.97, $\mathrm{p}=0.021$ ), gender $(\mathrm{OR}=0.53,95 \% \mathrm{CI}: 0.33-0.87 ; \mathrm{p}=0.011$ ), higher HbAlc level $(\mathrm{OR}=1.34,95 \% \mathrm{CI}: 1.13-1.60, \mathrm{p}=0.001)$, longer duration of diabetes (OR $=1.10,95 \% \mathrm{CI}: 1.07-1.14, \mathrm{p}<0.001)$, and insulin use ( $\mathrm{OR}=2.64,95 \% \mathrm{CI}$ : $1.50-4.64, \mathrm{p}=0.001)$ were independently associated with having any DR (Table 2). These five risk factors were therefore included in the CA for DR.

Only four factors were significantly associated with DME: younger 
Citation: Xie J, Fenwick EK, Taouk Y, Nicolaou TE, Wang JJ, et al. (2014) Relative Importance and Contribiton of Risk Factors for Diabetic Retinopathy and Macular Edema. J Diabetes Metab 5: 337. doi:10.4172/2155-6156.1000337

Page 3 of 7

\begin{tabular}{|c|c|c|c|c|c|}
\hline \multirow{3}{*}{\begin{tabular}{|l|} 
Characteristic \\
Categorical variable \\
\end{tabular}} & \multicolumn{4}{|c|}{ Diabetic Retinopathy $(n=497)$} & \multirow[b]{3}{*}{$\mathbf{p}$} \\
\hline & \multicolumn{2}{|c|}{ Absent $(n=200)$} & \multicolumn{2}{|c|}{ Present $(n=297)$} & \\
\hline & $\mathbf{N}^{\mathrm{a}}$ & $\%$ & $\mathbf{N}^{\mathrm{a}}$ & $\%$ & \\
\hline Gender (male) & 110 & 55.0 & 212 & 71.4 & $<0.001$ \\
\hline \multicolumn{6}{|l|}{ Income } \\
\hline$<\$ 30,000$ & 134 & 74.9 & 190 & 69.3 & \multirow{2}{*}{0.20} \\
\hline$\geq \$ 30,000$ & 45 & 25.1 & 84 & 30.7 & \\
\hline \multicolumn{6}{|l|}{ Education } \\
\hline Primary school or below & 32 & 16.4 & 42 & 14.6 & \multirow{3}{*}{0.62} \\
\hline Secondary school & 105 & 53.9 & 168 & 58.3 & \\
\hline 14 years or above & 58 & 29.7 & 78 & 27.1 & \\
\hline Current/past smoker & 113 & 57.4 & 156 & 53.1 & 0.35 \\
\hline Insulin use & 29 & 14.7 & 148 & 50.0 & $<0.001$ \\
\hline Comorbidities* $^{*}$ yes) & 178 & 89.0 & 248 & 83.5 & 0.09 \\
\hline Diabetic complication (yes) ${ }^{\Delta}$ & 44 & 22.0 & 116 & 39.1 & $<0.001$ \\
\hline Continuous variable & Mean/ median & SD/IQR & Mean/ median & SD/IQR & $\mathbf{p}$ \\
\hline Age (years)§ & 69.0 & 14.5 & 64.0 & 14.0 & $<0.001$ \\
\hline Systolic blood pressure, $\mathrm{mmHg}$ & 138.5 & 19.0 & 141.8 & 19.2 & 0.057 \\
\hline Diastolic blood pressure, $\mathrm{mmHg}$ & 77.0 & 8.2 & 75.9 & 9.1 & 0.17 \\
\hline Duration of diabetes (years) ${ }^{\S}$ & 8.0 & 9.0 & 16.3 & 13.0 & $<0.001$ \\
\hline BMI $\left(\mathrm{kg} / \mathrm{m}^{2}\right)$ & 30.6 & 6.7 & 31.4 & 6.2 & 0.25 \\
\hline Fasting plasma glucose, $\mathrm{mg} / \mathrm{dL}^{\S}$ & 7.0 & 2.4 & 8.4 & 7.8 & $<0.001$ \\
\hline $\mathrm{HbA} 1 \mathrm{c}(\%)^{\S}$ & 7.0 & 1.4 & 7.8 & 1.9 & $<0.001$ \\
\hline Total cholesterol, mmol/L§ & 4.6 & 1.5 & 4.3 & 1.5 & 0.18 \\
\hline HDL cholesterol, $\mathrm{mmol} / \mathrm{L}$ & 1.44 & 0.48 & 1.29 & 0.38 & $<0.001$ \\
\hline Triglycerides, mmol/L ${ }^{\S}$ & 1.5 & 0.9 & 1.6 & 1.2 & 0.49 \\
\hline LDL cholesterol, $\mathrm{mmol} / \mathrm{L}^{\S}$ & 2.2 & 1.2 & 2.2 & 1.2 & 0.86 \\
\hline
\end{tabular}

Data shown as medians (IQR) [§] for skewed data and mean (SD) for normally distributed data

*Includes hypertension, angina, irregular heartbeat, stroke, high cholesterol, asthma, anaemia, migraine, arthritis, and osteoporosis

$\Delta$ : nephropathy, peripheral vascular disease, neuropathy

avaries because of missing data. Bolded values indicate significant results

BMI: Body Mass Index; HDL: High-Density Lipoprotein; LDL: Low-Density Lipoprotein; SD: Standard Deviation; IQR: Interquartile Range. HbA1c: Haemoglobin A1c

Table 1: Patients' characteristics $(n=497)$ of those with and without diabetic retinopathy.

\begin{tabular}{|c|c|c|c|c|}
\hline \multirow{2}{*}{ Risk factor } & \multicolumn{2}{|c|}{$\mathrm{DR}^{\Delta}$} & \multicolumn{2}{|c|}{$\mathrm{DME}^{\Delta}$} \\
\hline & OR $(95 \% \mathrm{Cl})$ & p & OR $(95 \% \mathrm{Cl})$ & $\mathbf{p}$ \\
\hline Gender (female) & $0.53(0.33-0.87)$ & 0.011 & & \\
\hline Insulin use & $2.64(1.50-4.64)$ & 0.001 & & \\
\hline \multicolumn{5}{|l|}{ At least one comorbidity } \\
\hline \multicolumn{5}{|l|}{ Diabetic complication } \\
\hline Age (years) & $0.95(0.92-0.97)$ & $<0.001$ & $0.95(0.93,0.97)$ & $<0.001$ \\
\hline $\mathrm{SBP}, \mathrm{mmHg}$ & & & $1.01(1.00,1.02)$ & 0.092 \\
\hline Duration of diabetes (years) & $1.10(1.07-1.14)$ & $<0.001$ & $1.04(1.02,1.07)$ & $<0.001$ \\
\hline $\mathrm{HbA1c}(\%)$ & $1.34(1.13-1.60)$ & 0.001 & $1.41(1.20,1.67)$ & $<0.001$ \\
\hline
\end{tabular}

DR: Diabetic Retinopathy; DME: Diabetic Macular Edema; HbA1c: Haemoglobin A1c; SBP: Systolic Blood Pressure

$\triangle$ The independent variables include age, sex, body mass index (BMI), diabetes duration, smoking history, HbA1c, systolic blood pressure, total cholesterol, HDL-cholesterol, insulin use, other diabetic complication, fasting plasma glucose, and comorbidities

Table 2: Associations between risk factors for DR and DME in patients with type 2 diabetes from multiple logistic regression models.

age $(\mathrm{OR}=0.95,95 \%$ CI: 0.92-0.97, $\mathrm{p}=0.021)$, higher HbA1c level $(\mathrm{OR}$ $=1.34,95 \%$ CI: $1.13-1.60, \mathrm{p}=0.001)$, longer duration of diabetes $(\mathrm{OR}$ $=1.10,95 \%$ CI: $1.07-1.14, \mathrm{p}<0.001)$, and insulin use $(\mathrm{OR}=2.64,95 \%$ CI: $1.50-4.64, p=0.001)$ were independently associated with having any DME (Table 2). These four factors were therefore included in the CA for DME.

\section{Relative importance of key identified risk factors}

The commonality coefficients, which represent the proportion of variance in DR and DME explained by each risk factor uniquely and in combination, are shown in Tables 3 and 4, respectively. Overall, the commonality models indicate that these variables in total accounted for minimal to moderate proportions of variance in DME (19.48\%) and
DR (44.6\%), respectively. However, these values were still higher when compared to results obtained from multivariable logistic regression models (DME: $\mathrm{R}^{2}=12.77 \%$; and DR: $\mathrm{R}^{2}=26.14 \%$; Table 5).

There were also some important differences in the CA results between DR and DME. Of the five risk factors, duration of diabetes ( $8.7 \%$ of common variance and $7.9 \%$ of unique variance) was the largest contributor to the explained variance for DR. The unique and common contributions of $\mathrm{HbAlc}$ to DR were $2.1 \%$ and $6.4 \%$, respectively (Figure 1). However, HbAlc was the largest contributor (9.45\% in total: $5.41 \%$ of common variance and $4.04 \%$ of unique variance) to the explained variance of DME, followed by age, duration of DM and SBP (Figure 1).

Negative commonality coefficients indicate that two or more risk 
Citation: Xie J, Fenwick EK, Taouk Y, Nicolaou TE, Wang JJ, et al. (2014) Relative Importance and Contribiton of Risk Factors for Diabetic Retinopathy and Macular Edema. J Diabetes Metab 5: 337. doi:10.4172/2155-6156.1000337

Page 4 of 7

\section{Risk factor}

Unique to age

Unique to gender

Unique to $\mathrm{HbA} 1 \mathrm{c}$

Unique to insulin use

Unique to duration of diabetes

Common to age, and gender

Common to age, and $\mathrm{HbA} 1 \mathrm{c}$

Common to gender, and $\mathrm{HbA} 1 \mathrm{c}$

Common to age, and insulin use

Common to gender, and insulin use

Common to $\mathrm{HbA} 1 \mathrm{c}$, and insulin use

Common to age, and duration of diabetes

Common to gender and duration of diabetes

Common to $\mathrm{HbA} 1 \mathrm{c}$, and duration of diabetes

Common to insulin use and duration of diabetes

Common to age, gender, and $\mathrm{HbA} 1 \mathrm{c}$

Common to age, gender, and insulin use

Common to age, $\mathrm{HbA} 1 \mathrm{c}$, and insulin use

Common to gender, $\mathrm{HbA} 1 \mathrm{c}$, and insulin use

Common to age, gender, and duration of diabetes

Common to age, $\mathrm{HbA} 1 \mathrm{c}$, and duration of diabetes

Common to gender, $\mathrm{HbA} 1 \mathrm{c}$, and duration of diabetes

Common to age, insulin use, and duration of diabetes

Common to gender, insulin use, and duration of diabetes

Common to $\mathrm{HbA1c}$, insulin, and duration of diabetes

Common to age, gender, $\mathrm{HbA} 1 \mathrm{c}$, and insulin use

Common to age, gender, $\mathrm{HbA} 1 \mathrm{c}$, and duration of diabetes

Common to age, gender, insulin use, and duration of diabetes

Common to age, $\mathrm{HbA} 1 \mathrm{c}$, insulin use, and duration of diabetes

Common to gender, $\mathrm{HbA} 1 \mathrm{c}$, insulin use, and duration of diabetes

Common to age, gender, $\mathrm{HbA} 1 \mathrm{c}$, insulin use, and duration of diabetes

Total (\%)

commonality coefficient

3.20

1.51

2.12

2.79

7.85

0.50

2.29

$-0.15$

0.73

$-0.26$

0.38

$-1.85$

0.85

1.46

7.10

$-0.03$

$-0.01$

0.52

0.04

$-0.21$

$-0.64$

0.11

$-0.8$

0.25

2.29

0.00

0.07

0.01

$-0.12$

0.10

0.04

30.14
$\%$ Total

10.62

5.01

7.03

9.25

26.05

1.67

7.61

$-0.51$

2.41

$-0.87$

1.27

$-6.15$

2.83

4.86

23.54

$-0.09$

$-0.03$

1.74

0.14

$-0.70$

$-2.14$

0.37

$-2.65$

0.82

7.59

0.01

0.23

0.04

$-0.41$

0.33

0.14

100

Note: Commonality coefficient identifying suppression underlined. HbA1c: Hemoglobin (hemoglobinA1c). $\sum$ Commonality coefficient $=m u l t i p l e ~ R{ }^{2}(\%$ Total $=($ Commonality coefficient)/(multiple $R^{2}$ )

Table 3: Commonality matrix of risk factors for diabetic retinopathy (DR) in patients with type 2 diabetes.

\begin{tabular}{|l|c|}
\hline Risk factor & commonality coefficient \\
\hline Unique to age & 0.0386 \\
\hline Unique to SBP & 0.0046 \\
\hline Unique to HbA1c & 0.0404 \\
\hline Unique to duration of diabetes & 0.0358 \\
\hline Common to age and SBP & -0.0018 \\
\hline Common to age and HbA1c & 23.92 \\
\hline Common to SBP and HbA1c & -22.23 \\
\hline Common to age and duration of diabetes & -1.14 \\
\hline Common to SBP and duration of diabetes & 24.68 \\
\hline Common to HbA1c and duration of diabetes & 1.00 \\
\hline Common to age, SBP and HbA1c & -6.18 \\
\hline Common to age, SBP, and duration of diabetes & -0.01 \\
\hline Common to age, HbA1c, and duration of diabetes & 0.01 \\
\hline Common to SBP, HbA1c, and duration of diabetes & 0.00 \\
\hline Common to age, SBP, HbA1c, and duration of diabetes & -0.0216 \\
\hline Total $(\%)$ & -0.0011 \\
\hline
\end{tabular}

Note: Commonality coefficient identifying suppression underlined.

DME: Diabetic Macular Edema; HbA1c: Haemoglobin A1c; SBP: Systolic Blood Pressure

$\sum$ Commonality coefficient $=$ multiple $\mathrm{R}^{2}$

$\%$ Total= $($ Commonality coefficient $) /\left(\right.$ multiple $\left.R^{2}\right)$

Unique variance=x's unique variance; Common $=$ common variance; Total=Unique+Common;

Table 4: Commonality matrix of risk factors for diabetic macular edema (DME) in patients with type 2 diabetes. 
Citation: Xie J, Fenwick EK, Taouk Y, Nicolaou TE, Wang JJ, et al. (2014) Relative Importance and Contribiton of Risk Factors for Diabetic Retinopathy and Macular Edema. J Diabetes Metab 5: 337. doi:10.4172/2155-6156.1000337

Page 5 of 7

factors affected in opposition directions (Tables 3,4). For example, the most negative regression effect was confounded by the combinations of duration of diabetes and age $(-1.85 \%$, Table 3$)$, indicating that age was a protective factor and duration of diabetes was a risk factor for DR.

Overall, more than half $(27.1 / 44.6=60.8 \%)$ of the total explained variation of DR was shared among the five risk factors (Table 5). The largest common variance on DR is due to insulin use $(10.3 \%)$, representing the jointly effect with other factors. However, only $38.71 \%$ of the total explained variation of DME was shared variation among the four risk factors (Table 5).

Subgroup analysis showed the commonality models accounted for large amount of variation in VTDR (61.8\%) and PDR (63.50\%) (Table
S5). The variance of $\mathrm{HbA1c}$ was higher in patients with VTDR (unique variance: $3.5 \%$; common variance: $11.2 \%$ ) and PDR (unique variance: $3.4 \%$; common variance: $8.5 \%$ ), compared to that in patients with no DR.

\section{Discussion}

This study investigated the relative importance of major risk factors for DR and DME in a clinical cohort of adults with type 2 diabetes. The total variation; and independent and joint contributions of the key risk factors of DR and DME are markedly different. Our findings confirm that the ethology and pathogenesis of these two conditions are different and require prevention and intervention strategies. For example, based on our data, strategies for screening DR should prioritize knowing the
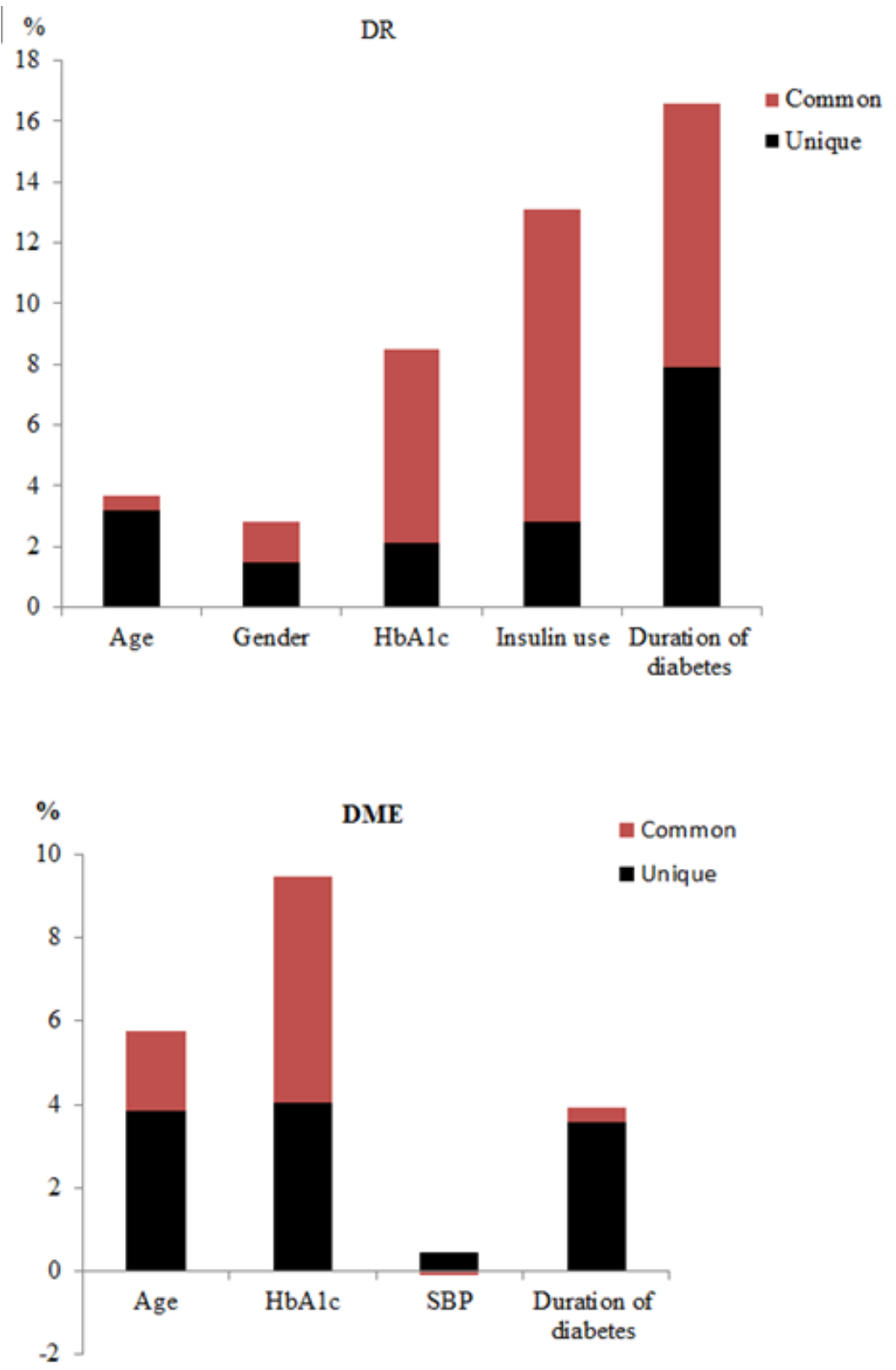

Figure 1: The unique and common variance (given as the percentage of the total explained variance) of the risk factors for Diabetic Retinopathy (DR) and Diabetic Macular Edema (DME) in patients with type 2 diabetes. 
Citation: Xie J, Fenwick EK, Taouk Y, Nicolaou TE, Wang JJ, et al. (2014) Relative Importance and Contribiton of Risk Factors for Diabetic Retinopathy and Macular Edema. J Diabetes Metab 5: 337. doi:10.4172/2155-6156.1000337

Page 6 of 7

\begin{tabular}{|c|c|c|c|c|c|c|}
\hline \multicolumn{7}{|l|}{ Outcome: DR } \\
\hline \multirow{2}{*}{ Risk factor (x) } & \multicolumn{3}{|c|}{ Logistic regression } & \multicolumn{3}{|c|}{ Commonality analysis } \\
\hline & OR & $\mathbf{p}$ & Adjusted R²(\%) & Unique (\%) & Common (\%) & Total (\%) \\
\hline Age & 0.95 & $<0.001$ & & 3.2 & 0.5 & 3.7 \\
\hline Gender & 0.53 & 0.005 & & 1.5 & 1.3 & 2.8 \\
\hline $\mathrm{HbA1c}$ & 1.34 & 0.001 & & 2.1 & 6.4 & 8.5 \\
\hline Insulin use & 2.64 & $<0.001$ & & 2.8 & 10.3 & 13.1 \\
\hline Duration of diabetes & 1.10 & $<0.001$ & & 7.9 & 8.7 & 16.5 \\
\hline Total & & & 26.14 & 17.5 & 27.1 & 44.6 \\
\hline \multicolumn{7}{|l|}{ Outcome: DME } \\
\hline \multirow{2}{*}{ Risk factor (x) } & \multicolumn{3}{|c|}{ Logistic regression } & \multicolumn{3}{|c|}{ Commonality analysis } \\
\hline & OR & $\mathbf{p}$ & Adjusted $\mathbf{R}^{2}(\%)$ & Unique (\%) & Common (\%) & Total (\%) \\
\hline Age & 0.95 & $<0.001$ & & 3.86 & 1.89 & 5.75 \\
\hline $\mathrm{HbA1c}$ & 0.66 & $<0.083$ & & 4.04 & 5.41 & 9.45 \\
\hline SBP & 1.41 & $<0.001$ & & 0.46 & -0.1 & 0.36 \\
\hline Duration of diabetes & 1.05 & $<0.001$ & & 3.58 & 0.34 & 3.92 \\
\hline Total & 0.95 & $<0.001$ & & 11.94 & 7.54 & 19.48 \\
\hline
\end{tabular}

Table 5: The results of the multiple logistic regression analysis and the commonality analysis for diabetic retinopathy (DR) and diabetic macular edema (DME).

duration of diabetes and the level of HbAlc. For DME, strategies for screening should prioritize the level of $\mathrm{HbAlc}$, the duration of diabetes and SBP.

While the results of this study are consistent with previous findings exploring individually the major risk factors for DR using logistic regression models $[3,15,24]$, the CA model provides additional information about the components of their regression effects. Because the pathogenesis of DR and DME is multi-factorial, identification of the relative importance of the major risk factors using traditional regression methods is difficult due to the collinearity present among explanatory variables, which may bias the importance of each individual risk factor. Furthermore, results tend to rely heavily on regression coefficients, which are not comparable directly due to different measurement scales.

The relative importance of the risk factors for DR and DME was evaluated by examining the proportion of unique and common variance using CA. Our study showed that the most important risk factors of DR are duration of diabetes, followed by insulin use, HbAlc, age, and gender. While it has been assumed that chronic hyperglycemiais critical to the development of DR, our study showed that $\mathrm{HbAlc}$ alone only contributed $2.1 \%$ of the total variance explained for DR. One previous study also reported that HbAlc and duration of diabetes explained only about $11 \%$ of the variation in risk of DR for the entire study population using traditional regression models, but this study did not include the common variance between these risk factors [10]. Blood pressure and HDL cholesterol were significantly associated with DR in the univariate analysis, but these associations disappeared in the adjusted model. Commonality analysis including SBP and HDL showed that those two factors explained a very small amount of variation in DR (online supplementary Table S2).

Our results from CA firstly showed that more than half of the total explained variation of DR was related to the joint variation between the five risk factors, which highlighted that multifactorial risk management approach to prevent DR is needed. On the other hand, we found that all those four risk factors in total explained a relative small proportion of total variance in DME by both logistic regression (12.77\%) and CA (19.48\%). SBP was significant associated with DME and contributed $0.4 \%$ of total explained variance. Importantly, our study showed that $\mathrm{HbA1c}$ is the primary risk factor for DME, which has strong clinical and public-health importance. On the other hand, the four independent risk factors for DME explains around 20\% of the variance, which suggesting that further research beyond the inclusion of traditional risk factors, is needed to understand the etiology and pathogenesis of DME.

The strengths of our study include the use of a novel statistical technique to demonstrate the relative importance of the main risk factors for DR and DME in a large clinical sample of patients with type 2 diabetes. The value of CA resides in the fact that the procedure yields unique and common variance explained from each of the risk factors. Our targeted study sample, patients of specialised eye clinics, may mean that our results are not generalizable to a wider population of patients with diabetes and DR/DME. Finally, we lacked sufficient power to explore the relative importance of risk factors for the severity of DR/ DME and the relative importance of risk factors for DR in patients with Type 1 diabetes, and further work is required to investigate this.

In conclusion, we demonstrated that the total variation; and independent and joint contributions of the key risk factors of DR and DME are markedly different in patients with type 2 diabetes. Our findings confirm that the ethology and pathogenesis of these two conditions are different and require prevention and intervention strategies.

\section{Financial support}

This study was supported by the National Health and Medical Research Council Centre for Clinical Research Excellence (CCRE) \#529923 - Translational Clinical Research in Major Eye Diseases; CCRE Diabetes; Australian Research Counci (ARC) Grant LP0884108; Royal Victorian Eye and Ear Hospital; Operational Infrastructure Support from the Victorian Government. The funding organizations had no role in the design or conduct of this research.

\section{Acknowledgments}

The authors thank their research collaborators, the Royal Victorian Eye and Ear Hospital (RVEEH) and their industry partner, Diabetes Australia, Victoria; for significant contributions to the design and implementation of the DMP; and all who agreed to participate in the DMP

\section{Contributors}

All authors contributed to the paper. JX undertook the analysis and prepared the manuscript. EF, JJW and TYW edited the paper. JX is guarantor of the data analysis. EL is the Principal Investigator and is overall guarantor for the whole study.

\section{Ethical Approval}

Ethical approval for the DMP was provided by the RVEEH Human Research and Ethics Committee $(08 / 815 \mathrm{H})$ 
Citation: Xie J, Fenwick EK, Taouk Y, Nicolaou TE, Wang JJ, et al. (2014) Relative Importance and Contribiton of Risk Factors for Diabetic Retinopathy and Macular Edema. J Diabetes Metab 5: 337. doi:10.4172/2155-6156.1000337

\section{References}

1. Yau JW, Rogers SL, Kawasaki R, Lamoureux EL, Kowalski JW, et al. (2012) Global prevalence and major risk factors of diabetic retinopathy. Diabetes Care 35: 556-564.

2. Shaw JE, Sicree RA, Zimmet PZ (2010) Global estimates of the prevalence of diabetes for 2010 and 2030. Diabetes Res Clin Pract 87: 4-14.

3. Klein BE (2007) Overview of epidemiologic studies of diabetic retinopathy. Ophthalmic Epidemiol 14: 179-183.

4. Wong T, Klein K (2008) The Epidemiology of Eye Diseases in Diabetes. In: Ekoé J, Rewers M, Williams R, Zimmet P (eds) The Epidemiology of Eye Diseases in Diabetes ( $2^{\text {nd }}$ edn) John Wiley and Sons, Oxford: 475-497.

5. Chatziralli IP, Sergentanis TN, Keryttopoulos P, Vatkalis N, Agorastos A, et al. (2010) Risk factors associated with diabetic retinopathy in patients with diabetes mellitus type 2. BMC Res Notes 3: 153.

6. Constable IJ, Knuiman MW, Welborn TA, Cooper RL, Stanton KM, et al. (1984) Assessing the risk of diabetic retinopathy. Am J Ophthalmol 97: 53-61.

7. Massin P, Lange C, Tichet J, Vol S, Erginay A, et al. (2011) Hemoglobin A1c and fasting plasma glucose levels as predictors of retinopathy at 10 years: the French DESIR study. Arch Ophthalmol 129: 188-195.

8. Control Group, Turnbull FM, Abraira C, Anderson RJ, Byington RP, et al. (2009) Intensive glucose control and macrovascular outcomes in type 2 diabetes. Diabetologia 52: 2288-2298.

9. Hirsch IB, Brownlee M (2010) Beyond hemoglobin A1c--need for additional markers of risk for diabetic microvascular complications. JAMA 303: 22912292.

10. Lachin JM, Genuth S, Nathan DM, Zinman B, Rutledge BN; DCCT/EDIC Research Group (2008) Effect of glycemic exposure on the risk of microvascular complications in the diabetes control and complications trial--revisited. Diabetes 57: 995-1001.

11. Case LD, Kimmick G, Paskett ED, Lohman K, Tucker R (2002) Interpreting measures of treatment effect in cancer clinical trials. Oncologist 7: 181-187.

12. Knol MJ, Duijnhoven RG, Grobbee DE, Moons KG, Groenwold RH (2011) Potential misinterpretation of treatment effects due to use of odds ratios and logistic regression in randomized controlled trials. PLoS One 6: e21248.
13. Service FJ, O'Brien PC (2001) The relation of glycaemia to the risk of development and progression of retinopathy in the Diabetic Control and Complications Trial. Diabetologia 44: 1215-1220.

14. Mohamed Q, Gillies MC, Wong TY (2007) Management of diabetic retinopathy: a systematic review. JAMA 298: 902-916.

15. Cheung N, Mitchell P, Wong TY (2010) Diabetic retinopathy. Lancet 376: 124 136

16. Nathans LL, Oswald FL, Nimon K (2012) Interpreting multiple linear regression A guidebook of variable importance. Practical, Assessment, Research, and Evaluation 17: 1-19.

17. Nimon K, Reio TG (2011) Regression Commonality Analysis: A Technique for Quantitative Theory Building. Human Resource Development Review 10: 329340 .

18. Reichwein Zientek L, Thompson B (2006) Commonality Analysis: Partitioning Variance to Facilitate Better Understanding of Data. Journal of Early Intervention 28: 299-307.

19. Nimon K, Lewis M, Kane R, Haynes RM (2008) An R package to compute commonality coefficients in the multiple regression case: an introduction to the package and a practical example. Behav Res Methods 40: 457-466.

20. Stellefson M, Yannessa JF, Martel GF (2012) Using canonical commonality analysis to examine the predictive quality of aging and falls efficacy on balance functioning in older adults. Eval Health Prof 35: 239-255.

21. Nimon KF, Oswald FL (2013) Understanding the Results of Multiple Linea Regression: Beyond Standardized Regression Coefficients. Organizational Research Methods.

22. Freberg ME, Vandiver BJ, Watkins MW, Canivez GL (2008) Significant factor score variability and the validity of the WISC-III Full Scale IQ in predicting later academic achievement. Appl Neuropsychol 15: 131-139.

23. Lamoureux EL, Fenwick E, Xie J, Mcauley A, Nicolaou T, et al. (2012) Methodology and early findings of the Diabetes Management Project: a cohort study investigating the barriers to optimal diabetes care in diabetic patients with and without diabetic retinopathy. Clin Experiment Ophthalmol 40: 73-82

24. Kloos C, Muller N, Wolf G, Hartmann P, Lehmann T, et al. (2011) Better HbA1c and blood pressure control in patients with diabetes mellitus treated at a primary health care level 10 years after initiation of a diabetes quality improvement program. Exp Clin Endocrinol Diabetes 119: 459-462.
This article was originally published in a special issue, Diabetic Retinopathy handled by Editor. Caroline J Magri, Mater Dei Hospital, Malta 